\title{
Etnografía de la comunicación en la educación en enfermería: perspectiva metodológica
}

Ethnography of communication in nursing education: methodological perspective Etnografia da comunicação na educação em enfermagem: perspectiva metodológica

José Luis Medina Moya ${ }^{1}$, Luz Nelly Rivera Álvarez², Jennifer Rojas Reyes $^{3}$

${ }^{1}$ PhD en Ciencias de la Educación, Enfermero, Pedagogo. Profesor titular. Universidad de Barcelona, Facultad de Pedagogía. Barcelona, España. Correo electrónico:jlmedina@ub.edu

${ }^{2} \mathrm{PhD}$ en Educación y Sociedad, Mg en Enfermería, Enfermera. Profesora asociada. Universidad Nacional de Colombia, Facultad de Enfermería. Bogotá, Colombia. Correo electrónico: Inriveraa@unal.edu.co

${ }^{3}$ Candidata a PhD en Enfermería, Mg en Enfermería, Enfermera. Financiada por la Convocatoria 727 de 2015 de Colciencias para Doctorados nacionales. Universidad Nacional de Colombia, Facultad de Enfermería. Bogotá, Colombia. Correo electrónico: jrojasre@unal.edu.co

Cómo citar este artículo en edición digital: Medina Moya, J.L., Rivera Álvarez, L.N. E Rojas Reyes, J. (2019). Etnografía de la comunicación en la educación en enfermería: perspectiva metodológica. Cultura de los Cuidados (Edición digital), 23(53). Recuperado de http://dx.doi.org/10.14198/cuid.2019.53.26

Correspondencia: Avenida Carrera 30 No. 45-03 Ciudad Universitaria Edificio 228, Piso 3 Of. 306.

Bogotá, Colombia

Correo electrónico: jrojasre@unal.edu.co

Recibido: 13/12/2018; Aceptado: 14/01/2019

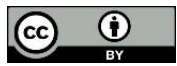

\section{ABSTRACT}

This review article and theoretical analysis is derived from multicentric research "Disciplinary knowledge, pedagogical knowledge and situated learning: genesis and mutual influences on university education", developed with Faculties of Health Sciences and Education in Universities of Spain, Brazil, Colombia and Chile. It aims to deepen the epistemological, theoretical and methodological perspective of the study as an innovative route in qualitative research, therefore, the epistemological foundations of Symbolic Interactionism are presented, which is based on the understanding of society through communication and allows to give meaning to human processes, such as the dialogical processes between teachers and students, analyzing the repercussions that it has on their learning. The theoretical perspective of the Ethnography of Communication that focuses on the analysis of the sociolinguistic patterns with which people interact, being a priority to know the meaning of verbal and non-verbal communication in this situation; and the microethnographic methodological perspective that achieves an understanding of the verbal and social interactions between teachers and students, through nonparticipant observations and "Think aloud" interviews. 
Keywords: Interdisciplinary research, education in nursing, ethnography of communication, qualitative research, symbolic interactionism.

\section{RESUMO}

Este artigo de revisão e análise teórica é derivado da pesquisa multicêntrica "conhecimento disciplinar, conhecimento e aprendizagem pedagógica implantada: génese e influências mútuas das universidades", desenvolvidos com faculdades de Ciências da Saúde e Educação em universidades em Espanha, Brasil, Colômbia e o Chile. Visa aprofundar a perspectiva epistemológica, teórica e metodológica do estudo como um caminho inovador na pesquisa qualitativa, portanto, são apresentados os fundamentos epistemológicos do Interacionismo Simbólico, que se baseia na compreensão da sociedade através da comunicação e permite dar sentido aos processos humanos, como os processos dialógicos entre professores e alunos, analisando as repercussões que tem sobre sua aprendizagem. A perspectiva teórica da Etnografia da Comunicação que foca a análise dos padrões sóciolingüísticos com os quais as pessoas interagem, sendo uma prioridade conhecer o significado da comunicação verbal e não-verbal nessa situação; e a perspectiva metodológica microetnográfica que alcança uma compreensão das interações verbais e sociais entre professores e alunos, através de observações não-participantes e Pense em voz alta em entrevistas.

Palavras-chave: Pesquisa interdisciplinar, educação em enfermagem, etnografia da comunicação, pesquisa qualitativa, interacionismo simbólico.

\section{RESUMEN}

Este artículo de revisión y análisis teórico se deriva de la investigación multicéntrica "Saberes disciplinares, saberes pedagógicos y aprendizaje situado: génesis e influencias mutuas en la enseñanza universitaria", desarrollada con facultades de Ciencias de la Salud y de la Educación en Universidades de España, Brasil, Colombia y Chile. Pretende profundizar la perspectiva epistemológica, teórica y metodológica del estudio como una ruta innovadora en la investigación cualitativa, por tanto, se presentan los fundamentos epistemológicos del Interaccionismo Simbólico, que se basa en la comprensión de la sociedad a través de la comunicación y permite dar significado a procesos humanos, como los procesos dialógicos entre profesores y estudiantes, analizando las repercusiones que tiene en su aprendizaje. La perspectiva teórica de la Etnografía de la Comunicación que se centra en el análisis de los patrones sociolingüísticos con los que interactúan las personas, siendo prioritario conocer el significado que posee la comunicación verbal y no verbal en esta situación; y la perspectiva metodológica microetnográfica que logra una comprensión de las interacciones verbales $y$ sociales entre profesores y estudiantes, por medio de observaciones no participantes y entrevistas "Think aloud".

Palabras clave: Investigación interdisciplinaria, educación en enfermería, etnografía de la comunicación, investigación cualitativa, interaccionismo simbólico.

\section{INTRODUCCIÓN}

La evolución de la investigación en enfermería, ha abierto las puertas a diversos métodos de indagación y también a la expansión hacia otras temáticas que abordan aspectos interdisciplinares, los 
cuales le han permitido conocer las necesidades en cada uno de sus roles (Arias y Gaviria, 2014). Es así como la investigación en educación aporta elementos que permiten el entendimiento de los procesos de pedagógicos en enfermería, basándose en conceptos y teorías de ambas disciplinas. Pretender conocer y comprender las características de la cultura educativa en enfermería, implica dar un avance en la investigación interdisciplinaria con métodos innovadores.

Una perspectiva investigativa compartida entre la educación y la enfermería frente a aspectos culturales en la formación es la etnografía. Ésta hace parte del abordaje cualitativo y se ha orientado al estudio de las costumbres y tradiciones de las comunidades, hace la descripción e interpretación del comportamiento cultural en el que se puede contemplar un grupo humano en particular, en situaciones igualmente particulares (Moncada y Pinilla, 2006). De allí que la etnografía haya tomado diferentes caminos de acuerdo a los contextos y necesidades investigativas como la etno-enfermería, la etnografía de la comunicación, la etnografía de la educación, la etnografía de procesos, entre otras.

En este caso, se expondrá el uso de la etnografía de la comunicación en los procesos de enseñanza y aprendizaje en enfermería, contribuyendo al descubrimiento de las complejidades que encierran los fenómenos educativos en una ciencia como enfermería (Polifroni, 2011), la cual tiene un alto contenido de responsabilidad social por el cuidado de las personas. La enseñanza y aprendizaje de esta disciplina implica un adecuado conocimiento didáctico del contendido del docente, además de estrategias prácticas que lleven al estudiante a comprender la importancia del conocimiento que va a adquirir, contribuyendo al descubrimiento de las complejidades comunicativas que encierran los fenómenos educativos en enfermería (Shulman, 1987; Medina y Jarauta, 2013).

Basados en el desarrollo del proyecto multicéntrico "Saberes disciplinares, saberes pedagógicos y aprendizaje situado: génesis e influencias mutuas en la enseñanza universitaria", el cual propone entender las interacciones entre los saberes disciplinares y pedagógicos del docente y los aprendizajes de los estudiantes de enfermería en las aulas. Explicitando esto, se sabe que las respuestas que los profesores ofrecen a las contribuciones de los estudiantes durante sus interacciones en el aula suponen un proceso complejo (Medina, Rivera y Carrillo, 2015), que desde el punto de vista sociolingüístico, acontece porque el docente identifica en las contribuciones de los estudiantes marcadores de reconocimiento y a partir de ellos interpreta, organiza y elabora la respuesta que emitirá.

Por lo anterior, se hace relevante describir y analizar teóricamente el diseño metodológico que se sigue en esta investigación y que se presenta como alternativa a otras rutas comunes en el abordaje cualitativo, explorando la etnografía de la comunicación en un ambiente educativo pero disciplinarmente determinado. Se expone la base epistemológica, teórica y metodológica que concuerdan con el estudio en mención.

\section{PERSPECTIVA EPISTEMOLÓGICA}

Los fundamentos filosóficos y/o epistemológicos dan la base para orientar la perspectiva en la que se enfocan los objetivos de investigación y sus resultados. La forma en que se obtiene o construye el conocimiento es el punto que permite 
diferenciar los caminos para llegar a conocer un fenómeno y éste conduce a un método de indagación específico.

En este caso, la base epistemológica del proyecto multicéntrico es el Interaccionismo simbólico justificado en la naturaleza social, simbólica y no lineal del objeto de estudio. Esta es una corriente del pensamiento que se basa en la comprensión de la sociedad a través de la comunicación, situado en el paradigma de la transmisión de la información: emisor-mensaje-receptor, donde se considera a la comunicación como un hecho instrumental (Blummer, 1969).

Podría parecer confuso que la base epistemológica de la etnografía de la comunicación sea el interaccionismo simbólico, en especial porque la etnografía tiene su origen en la antropología y el interaccionismo surge desde la sociología, sin embargo, es así, a razón de que las vertientes etnográficas que surgieron respondieron a lo cultural y contextual pero no a la interpretación simbólica. Tal es el caso de este diseño de investigación, que centra su análisis en cuestiones sociolingüísticas obligando a interpretar los símbolos que de la interacción se generan, $y$ es allí donde juega un papel muy importante los principios del interaccionismo como la forma de construir conocimiento.

El interaccionismo simbólico enfatiza la importancia del significado e interpretación en los procesos humanos esenciales. En educación, los procesos de enseñanza y los del aprendizaje se concretan mediante el uso de lenguajes y representaciones, accionados en un contexto sociocultural que presenta construcciones simbólicas acordes con fines específicos (Shablico, 2012).

Es así que, el significado de una conducta se forma en la interacción social, que da como resultado un sistema de significados intersubjetivos y el contenido del significado no es más que la reacción de los actores ante la acción en cuestión (Morse, 2003), siendo así, se deben considerar los principios bajo los cuales se rige (Blummer, 1969; Mead, 1972):

1. A diferencia de los animales, los seres humanos están dotados de capacidad de pensamiento.

2. La capacidad de pensamiento está modelada por la interacción social.

3. En la interacción social las personas aprenden los significados y los símbolos que les permiten ejercer su capacidad de pensamiento.

4. Los significados y los símbolos permiten a las personas actuar e interactuar de una manera distintivamente humana.

5. Las personas son capaces de modificar o alterar los significados y los símbolos que usan en la acción y la interacción sobre la base de su interpretación de la situación.

Blumer (1969) resume el interaccionismo simbólico en tres premisas simples. La primera es que las personas actúan en relación a las cosas a partir del significado que le asignan. La segunda dice que el contenido de las cosas se define a partir de la interacción social que el individuo tiene con los demás. El tercero implica que el contenido es trabajado y modificado a través de un proceso de traducción y evaluación que el individuo usa cuando trabaja las cosas con las que se encuentra.

La importancia del interaccionismo simbólico para la investigación cualitativa es su énfasis distintivo sobre la importancia de símbolos y lo fundamental de los procesos interpretativos generados en base a interacciones, para entender la conducta humana. Desde esta perspectiva, se afirma que la naturaleza de los procesos de 
enseñanza-aprendizaje sólo puede ser desentrañada mediante su examen directo, permitiendo construir e interpretar la forma en que los procesos dialógicos mediante los que el profesor acopla sus percepciones y significaciones con las de los estudiantes tiene repercusiones en su aprendizaje (Bautista, 2011).

\section{PERSPECTIVA TEÓRICA}

Las aulas se han convertido en lugares de socialización para estudiantes de diversos orígenes étnicos y culturales, en consecuencia se hace necesario el conocimiento y comprensión de estos para orientar la práctica pedagógica en general; sin embargo, no se debe desconocer que los docentes también constituyen grupos de culturas específicas, que a su vez deben dialogar con otras. La interacción entre docentes y estudiantes, el descubrimiento de modelos educativos, el análisis del currículo oculto y el análisis de contextos educativos, constituyen algunas temáticas abordables desde el enfoque etnográfico (Sandin, 2003).

Teóricamente, el proyecto multicéntrico se enmarca en las bases de la Etnografía de la comunicación, la cual se centra en el análisis de los patrones sociolingüísticos con los que interactúan los miembros de una comunidad. Estos aspectos se asumen desde la comunicación verbal y no verbal, y ambas son culturalmente modeladas, aunque las personas en interacción no tengan conciencia de ello (Álvarez, 2008). Hymes, es el mayor representante de la etnografía de la comunicación, quien la definió como una composición teórica orientada a aprehender los datos del contexto asociados al lenguaje (Prado, 2013).

Diversos autores consideran que, comunicarse implica participar en una forma de comportamiento determinado por pautas relativamente estables y sistemáticas; pero estas pautas no son sólo de naturaleza gramatical, sino también de naturaleza pragmática que orienta cognitiva $y$ socialmente el acto comunicativo (Escandel, 2010; Bausells, 2013).

En el mismo sentido, aparece la comunicación no verbal en la interacción, siendo incluso más relevante que la verbal porque permite enfatizar y reforzar los mensajes lingüísticos. A nivel educativo, en las relaciones docente-estudiante logra transmitir las actitudes del docente hacia los contenidos y los estudiantes, su ideología, sus expectativas hacia el grupo en general y a cada uno en particular; a su vez, influye sobre la percepción que el alumno tiene del profesor y de la materia y sobre su grado de satisfacción (Álvarez, 2012).

Esta investigación entre España, Brasil, Colombia y Chile pretende profundizar en el conocimiento de los procesos dialógicos mediante los que el profesor acopla sus percepciones y significaciones con las de los estudiantes y sus repercusiones en el aprendizaje, avanzando en la comprensión de los procesos de coordinación entre los saberes disciplinares y pedagógicos del docente y el aprendizaje del estudiante (Medina et al., 2015).

Lo anterior, se acoge a los principios de la etnografía de la comunicación, porque describe cómo el profesor percibe las contribuciones del estudiante, cómo las interpreta, evalúa y discrimina en ellas lo relevante, haciendo evidente aspectos verbales y no verbales; además, al mostrar cómo responde el docente a los estudiantes y describir su razonamiento y acción pedagógica se manifiestan los procesos lingüísticos, sociales y cognitivos que pueden ser estudiados por este método.

Las formas comunicativas dentro de la educación en enfermería junto con el 
conocimiento didáctico del contenido disciplinar, deben ser vistos como símbolos culturalmente aceptados que se convierten en un lenguaje común entre profesores y estudiantes de enfermería, pero que merecen ser interpretados porque podrían no ser comunes a todas las comunidades educativas de disciplinas similares (Medina et al., 2015).

Como diseño de investigación, la etnografía de la comunicación también permite una aproximación desde la intersubjetividad, que toma como punto de partida los usos del lenguaje en los contextos sociales del aula y su relación con las dinámicas de significación y de aprendizaje en la experiencia inmediata de profesores y estudiantes (con énfasis en las del docente) que, cronológicamente, pueden durar segundos y que desde un punto de vista sociocultural es cuando acontece el aprendizaje (Duranti, 1992; Vygotsky, 2010).

EI interés por el uso del lenguaje no es entonces, únicamente, un compromiso metodológico para poder responder a la pregunta de qué es lo que dicen las personas en una variedad de contextos, sino, además, conocer lo que hacen con el lenguaje, voluntaria o involuntariamente (Hymes, 1962). En concreto, una de las razones que aconsejan su elección como punto de partida de la investigación es la necesidad de rechazar la convicción de que compartir la misma "lengua" implica un conocimiento también compartido del uso y del significado en diversos contextos (Hymes, 1962).

Conforme a lo descrito, Shablico (2010) argumenta que el lenguaje y las conductas no verbales del profesor y los estudiantes, son mediadores para el intercambio de información, símbolos y significados que promueven nuevos procesos mentales, y que esta dinámica está inmersa en una cultura $\mathrm{y}$ un pensamiento colectivo e individual que tienen en cuenta el habla, la entonación de las palabras, la mirada, el silencio, la gestualidad y los movimientos corporales como aspectos indispensables en el entendimiento del otro.

Así, con la etnografía de la comunicación se tiende a iniciar el análisis del comportamiento lingüístico, por lo que, el habla o su ausencia, parecen ser significativas en la mayoría de las interacciones humanas. Su contribución teórica fundamental, más allá de la descripción de modelos comunicativos, consiste en el análisis del papel que desempeña el habla en el moldeamiento de la vida de las personas (Sandin, 2003; Gumperz, 1982; Pardo, 1998) y esto es ratificado en el desarrollo del proyecto multicéntrico.

\section{PERSPECTIVA METODOLÓGICA}

Metodológicamente, el proyecto multicéntrico desarrolla un acercamiento microetnográfico, basado en la sociolingüística en su vertiente microsocial (Gumperz, 1982). La Microetnografía consiste en focalizar el trabajo de campo a través de la observación e interpretación del fenómeno en una sola institución social, en una o varias situaciones sociales (Atkinson y Hammersley, 1994). En educación, este enfoque se centra en la situación de interacción docente-estudiante al interior del aula de clase, tratando de demostrar que la interacción verbal y no verbal, no sólo es determinante en el éxito o el fracaso académico de los estudiantes, sino que a su vez depende, en su naturaleza y contenido, de la "competencia comunicativa" existente entre los actores (Zubieta, 1982).

El principal aporte de este tipo de método ha sido el de enriquecer los análisis de los 
hechos educativos con la contrastación entre la norma y la realidad del aula de clase, en la que los resultados de la micro-etnografía son de gran utilidad para implementar reformas que no comprometen la estructura social, pero que sí han representado una orientación valiosa para la labor docente (Zubieta, 1982).
Para hacer operativa la microetnografía, se deben usar un conjunto de herramientas intelectuales y de técnicas de recogida (Weston y McAlpine, 2002; Erickson y Simon, 1993), análisis e interpretación de la información, las cuales se explican en la tabla 1 y fueron utilizadas en el proyecto multicéntrico.

\section{TABLA 1: Técnicas de recolección}

\begin{tabular}{|c|c|}
\hline TÉCNICA DE RECOLECCIÓN & DESCRIPCIÓN \\
\hline $\begin{array}{l}\text { Observación persistente-no participante. } \\
\text { (Kawulich, 2006; Valles, 1999) }\end{array}$ & $\begin{array}{l}\text { La observación persistente, permite verificar cambios a través } \\
\text { del tiempo en eventos ya observados. Esta forma de } \\
\text { observación requiere de muchas sesiones para recabar } \\
\text { información relevante. También se desarrollará la observación } \\
\text { no participante (Sandoval, 1997) en la que está presente el } \\
\text { observador, pero no interactúa, ya que se hace indispensable } \\
\text { mantener la objetividad en el proceso y evitar inducir acciones } \\
\text { en las actividades que se observen (Bautista, 2011). }\end{array}$ \\
\hline $\begin{array}{l}\text { Técnica de las transcripciones paralelas. } \\
\text { (Velasco, 1999) }\end{array}$ & $\begin{array}{l}\text { Esta técnica permite documentar cómo diferentes individuos } \\
\text { interpretan y responden a una misma experiencia en la que } \\
\text { participan. El objetivo de esta es contrastar un mismo evento } \\
\text { desde diversos puntos de vista de los participantes y del mismo } \\
\text { investigador. Permite comprobar la interpretación común y } \\
\text { diferenciada que dan a las situaciones estudiadas, lo que } \\
\text { enriquece el análisis de los datos al ser presentados de forma } \\
\text { comparativa (Weston y McAlpine, 2002). }\end{array}$ \\
\hline $\begin{array}{l}\text { Entrevistas biográficas. } \\
\text { (Valles, 1999) }\end{array}$ & $\begin{array}{l}\text { En los relatos biográficos, el entrevistado tiende a dar tanta } \\
\text { información como sea necesario para poder mostrar la } \\
\text { transición y vínculos entre unos acontecimientos y otros. Se } \\
\text { dan detalles sobre el tiempo, los espacios, los motivos y otros } \\
\text { aspectos en los que se deben identificar los acontecimientos de } \\
\text { mayor importancia para el fenómeno estudiado. Este tipo de } \\
\text { entrevista permite entender cómo los acontecimientos fueron } \\
\text { vividos e influyeron en las acciones tomadas. }\end{array}$ \\
\hline $\begin{array}{l}\text { Entrevistas de "Think aloud". } \\
\text { (Charters, 2003) }\end{array}$ & $\begin{array}{l}\text { Proporcionan una visión de los procesos cognitivos del } \\
\text { participante, haciendo explícito su pensamiento durante el } \\
\text { desempeño de las tareas. En su versión original, esta técnica } \\
\text { invita al participante a verbalizar sus acciones mientras las } \\
\text { desarrolla (Erickson y Simon, 1993). Aquí se aplica un } \\
\text { protocolo retrospectivo, recogido después de la tarea, donde el } \\
\text { participante retrocede sobre lo que hizo, motivado por una } \\
\text { grabación de vídeo de sí mismo, porque en el momento en que } \\
\text { se da el evento no es posible interrumpir la acción (Fonteyn, } \\
\text { Kuipers y Grobe, 1993). }\end{array}$ \\
\hline
\end{tabular}

FUENTE: Elaboración de los autores 
Partiendo de la premisa sociolingüística de que la construcción de sentidos depende de las diversas formas que adopta la combinación de contenidos y significados durante el flujo del discurso, es de esperarse que el proceso de construcción de significados no sea un asunto únicamente de contenidos sino de combinación secuencial de contenidos (Nitti, Ciavolino, Salvatore y Gennaro, 2010). Por ende, en lo que respecta al análisis de datos, se utilizan conjuntamente estrategias como las comparaciones constantes (Charmaz, 2006; Corbin y Straus, 2002) y el Análisis Secuencial del Flujo del Discurso (Nitti et al., 2010).

Frente a la comparación constante (Charmaz, 2006; Corbin y Straus, 2002) la recolección, codificación y análisis de datos se hace de forma sistemática, contrastando similitudes y diferencias de los datos con el objetivo de descubrir patrones. Por otro lado, el Análisis Secuencial del Flujo del
Discurso consiste en la construcción de sentidos centrándose en los de patrones temporales de significación y que permite analizar la construcción intra e intersubjetiva de sentido con el fin de ir más allá de los límites del análisis semántico del discurso (Nitti et al., 2010).

En la fase de microanálisis del proyecto multicéntrico, se tiene en cuenta la unidad de análisis conformada por la interacción EP-E' (estudiante-profesor-estudiante), en la que se inicia con la contribución del estudiante, luego el profesor responde de forma inmediata y se observa cómo esta respuesta es recibida por el estudiante. El enfoque microanalítico se caracteriza por exigir altos grados de minuciosidad para tal efecto se descompondrá la intervención del profesor (P) en tres momentos (identificación-evaluación-respuesta) que darán como resultado la unidad ampliada E$\mathrm{P}$ (i-e-r)-E', como se presenta en la siguiente tabla:

TABLA 2: Análisis por elementos de la Unidad E- P (i-e-r)-E'

\begin{tabular}{|c|c|c|}
\hline \multicolumn{2}{|c|}{$\begin{array}{l}\text { Unidad de análisis } \\
\text { E-P(i-e-r)-E }\end{array}$} & Tipo de análisis y elemento de la unidad analizado \\
\hline Alumno & Contribución & $\begin{array}{l}\text { Análisis del discurso-Elemento E: Identificación inductiva de la } \\
\text { tipología de contribuciones del estudiante a las que el profesor } \\
\text { responde. }\end{array}$ \\
\hline \multirow[b]{3}{*}{ Profesor } & Identificación & \multirow{2}{*}{$\begin{array}{l}\text { Análisis del discurso-Elementos E-P(i-e): A partir del relato } \\
\text { introspectivo del profesor sobre su acción se reconstruyen los } \\
\text { microeventos de percepción, descripción, apreciación, } \\
\text { categorización y significación de la contribución del estudiante. }\end{array}$} \\
\hline & Evaluación & \\
\hline & Respuesta & $\begin{array}{l}\text { Análisis del discurso-Elementos } P(e-r) \text { : A partir del relato } \\
\text { introspectivo del profesor sobre su acción se reconstruyen los } \\
\text { microeventos que explican el progresivo despliegue de la } \\
\text { elaboración de la respuesta. } \\
\text { Análisis del discurso-Elemento } P(r) \text { : Identificación inductiva de la } \\
\text { tipología de respuestas del profesor. }\end{array}$ \\
\hline Alumno & Contribución & $\begin{array}{l}\text { Análisis del discurso-Elemento } R-E^{\prime} \text { : Identificación inductiva de la } \\
\text { tipología de las reacciones del estudiante a la respuesta del profesor. } \\
\text { Análisis Elementos } E-P(r)-E^{\prime} \text { : Comparación de los estados } \\
\text { cognitivos del estudiante (atencionales, perceptuales, conceptuales) } \\
\text { antes y después de la respuesta del profesor. }\end{array}$ \\
\hline
\end{tabular}


Una vez realizado el microanálisis de todos los datos, se llevará a cabo un análisis relacional y contextualizado que los integre y permita describir los procesos de pensamiento y acción pedagógica que contribuyen efectivamente al desarrollo de aprendizajes de alta calidad y además construir modelos de práctica docente dialógica que basados en ellos. Estos resultados serán triangulados con los profesores participantes y con los especialistas internacionales del equipo de trabajo (Medina, 2014; Taylor y Bodgan, 1987).

\section{CONCLUSIÓN}

La etnografía de la comunicación tiene sus bases de conocimiento en el interaccionismo simbólico y se concreta metodológicamente en la microetnografía con diferentes estrategias de recolección y análisis características de la investigación cualitativa. Esta es una ruta propuesta para la obtención de conocimiento dialógico en las interacciones docente-estudiante en el aula, lo que implica un diseño con raíces sociales y antropológicas que no compiten entre ellas sino que se nutren de sus bases.

Con esto es importante reflexionar sobre la flexibilidad en la investigación cualitativa, en la existen múltiples formas de abordar un fenómeno, sin dejar de lado, el rigor, la coherencia interna. Esto se logra al demostrar la conexión epistemológica, teórica y metodológica que fundamentan el desarrollo de una investigación.

El proyecto multicéntrico utilizado como ejemplo de esta ruta metodológica permite reconocer la importancia de la reflexión del docente durante su proceso de enseñanza, porque contribuye a un aprendizaje significativo del estudiante, de modo que, comprender este proceso dialógico dentro del aula en enfermería, apoya desarrollo de pedagogías innovadoras en los programas curriculares y otros proyectos de investigación interdisciplinares e interinstitucionales enfocados en dar respuestas pertinentes en lo académico y lo social.

Aclaraciones: Proyecto financiado en Colombia por la Dirección de Investigación y Extensión de la sede Bogotá (DIEB) de la Universidad Nacional de Colombia. CODIGO HERMES: 34917 y por el Ministerio de Educación de España a través del Grupo de investigación de Formación Docente e Innovación Pedagógica FODIP (2104SGR-335).

Conflicto de intereses: Los autores no tiene ningún tipo de conflicto de intereses.

\section{REFERENCIAS BIBLIOGRÁFICAS}

- Álvarez Núñez, Q. (2012). La comunicación no verbal en los procesos de enseñanza-aprendizaje: el papel del profesor. Innovación Educativa, (22), 23-37. - Álvarez, C. (2008). Ethnography as a research model in education. Gazeta de Antropología, 24(1), artículo $10 . \quad$ Disponible en: http://hdl.handle.net/10481/6998.

- Arias Valencia, MM. y Gaviria Noreña, DL. (2014) La investigación cualitativa: un acto creativo por la enfermería. Av.enferm., XXXII (2), 280-291.

- Atkinson, P. y Hammersley, M. (1994). Etnografía. Métodos de investigación. Barcelona: Paidós Básica.

- Bausells Espin, A. (2013). El factor socio-pragmático en el proceso de enseñanza aprendizaje de ELE (Tesis de Maestría). Universidad de Alcalá, Madrid, España. - Bautista, NP. (2011). Proceso de la investigación cualitativa. Epistemología, metodología y aplicaciones. Bogotá, Colombia: Manual Moderno.

- Blumer, H. (1969). Symbolic Interactionism. Perspective and method. University of California Press.

- Charmaz, K. (2006). Constructing Grounded Theory. London: SAGE publications.

- Charters, E. (2003). The use of Think-aloud methods in qualitative research: An introduction to Think-aloud Methods. Brock Education, 12(2), 68-82. 
- Corbin, J., y Straus, A. (2002). Bases de la investigación cualitativa: Técnicas y procedimientos para desarrollar la teoría fundamentada. Antioquia, Colombia: Editorial Universidad de Antioquía.

- Duranti, A. (1992). La etnografía del habla: hacia una lingüística de la praxis En FJ. Newmeyer (ed.) Panorama de la lingüística moderna de la Universidad de Cambridge. Vol. 4. El lenguaje: contexto sociocultural. (pp.252-269). España: CSUC.

- Erickson, K., y Simon, H. (1993). Protocol Analysis: Verbal Reports as Data. Boston: MIT Press.

- Escandel Vidal, MV. (2010). La Pragmática en la enseñanza de lenguas extranjeras. En J. Barrio (Coordinador), La pragmática en la enseñanza. Mesa redonda llevada a cabo en las Jornadas de formación del profesorado en la enseñanza de L2/ELE y la Literatura Española Contemporánea, Universidad de Sofía "San Clemente de Ojrid", Bulgaria.

- Fonteyn, M., Kuipers, B., y Grobe, S. (1993). A description of think aloud method and protocol analysis. Qualitative Health Research, 3(4), 430-441.

- Gumperz, J. (1982). Discourse Strategies. Oxford, U.K.: Oxford University Press.

- Hymes, D. (1962). The ethnography of speaking. En T. Gladwin, W. Sturtevant \& A. and Education (Eds.), Anthropology and Education. Washington, DC: Anthropological Society of Washington.

- Kawulich, B. (2006). La observación participante como método de recolección de datos. Forum: Qualitative Social Research, 6(2), Art. 43. Disponible en: www.qualitative-

research.net/index.php/fqs/article/download/466/999.

- Mead, GH. (1972). Espíritu, persona y sociedad: desde el punto de vista del conductivismo social. Buenos Aires: Paidós.

- Medina Moya, J. (2014). El proceso de comprensión en el análisis de datos cualitativos en educación. Magis. Revista internacional de investigación en Educación, 7(14), 39-54.

- Medina, JL., Rivera, LN., Carrillo, M. (2015). Saberes disciplinares, saberes pedagógicos $\mathrm{y}$ aprendizaje situado: génesis e influencias mutuas en la enseñanza universitaria-Colombia. Formación Docente e Innovación Pedagógica FODIP (2104SGR-335). Barcelona.

- Medina, JL., y Jarauta, B. (2013). Análisis del Conocimiento Didáctico del Contenido de tres profesores universitarios. Revista de Educación, (360), 600-623.

- Moncada, LI. y Pinilla, AE. (2006). Research and education in the medicine school. Rev Fac Med Univ Nac Colomb., 54, 313-329.

- Morse, J. (ed.). (2003). Asuntos críticos en la metodología de investigación cualitativa. Colombia:
Universidad de Antioquía.

- Nitti, M., Ciavolino, E., Salvatore, S., y Gennaro, A. (2010). Analyzing psychotherapy process as intersubjective sensemaking: an approach based on discourse analysis and neural networks. Psychotherapy Research: Journal of the Society for Psychotherapy Research, 20(5), 546-563.

- Pardo Abril, NG. (1998). Etnografía del habla: una perspectiva del análisis del lenguaje. Forma y Función, (11), 149-160.

- Polifroni, C. (2011). Philosophy of science: an introduction. En J. Butts and K. Rich (ed.), Philosophies and theories for advanced nursing (pp. 218). Salsburry, Mass: Jones and Bartlet.

- Prado Ballester, C. (2007). La Etnografía de la Comunicación. Un Modelo Olvidado. VI Congreso Chileno de Antropología. Colegio de Antropólogos de Chile A. G, Valdivia.

- Sandin, MP. (2003). Capítulo 7. Tradiciones en la investigación cualitativa en educación. En Fundamentos y Tradiciones. Madrid, España: McGraw Hill Interamericana de España.

- Sandoval, C. (1997). Métodos y técnicas avanzadas de investigaciones aplicadas a la educación y a las ciencias. Módulo 4: Investigación Cualitativa. Bogotá: ICFES.

- Shablico, S. (2012). La comunicación no verbal en el aula, un análisis en la enseñanza disciplinar. Cuadernos de Investigación Educativa, 3(18), 99-121.

- Shulman, L. (1987). Knowledge and teaching: foundations of the New Reform. Harvard Educational Review, 57(1), 1-22.

- Taylor, SJ., y Bogdan, R. (1987). Introducción a los métodos cualitativos de investigación. Barcelona, España: Paidós.

- Valles, M. (1999). Técnicas cualitativas de investigación social. Madrid, España: Síntesis S.A.

- Velasco, MC. (1999). El método biográfico y las historias de vida. Su utilidad en la investigación en enfermería. Índex de enfermería, 27, 25-30. Disponible en: http://www.index-f.com/indexenfermeria/27revista/27_articulo_25-30.php.

- Vygotsky, L. (2010). Pensamiento y lenguaje. Barcelona: Paidós.

- Weston, C., y McAlpine, L. (2002). Parallel transcripts: an innovative approach for capturing the impact of reflective teaching on student learning experience. In Annual Meeting of the American Education Research Association. New Orleans, LA.

- Zubieta, L. (1982). Etnografía y Política Educativa. Ponencia presentada al Seminario de Administración Educativa. Universidad Pedagógica Nacional. Bogotá: REDACADEMICA. 